\title{
Die Verwendung des nominativischen und illativischen Infinitivs im Mordwinischen
}

In Mordwinischen gibt es zwei Infinitive, die nebeneinander und in den gleichen Funktionen verwendet werden wie der eine Infinitiv der indoeuropäischen Sprachen. Im Ostseefinnischen entsprechen ihnen ebenfalls zwei Infinitive, der 1. Infinitiv und der Illativ des 3. Infinitivs. Die mordwinischen Infinitive enthalten ein Zeichen mit einem $m$; dieser Charakter ist identisch mit dem -ma, -mä des ostseefinnischen 3. Infinitivs. Der eine hat die Lautgestalt des Nominativs, der andere ist die mit der Illativendung $s$ gebildete Kasusform. Das Kennzeichen des nominativischen Infinitivs ist im Ersamordwinischen in hintervokalischen Wörtern -mo, in vordervokalischen -me und in Mokšamordwinischen entsprechend $-m \check{a},-m \check{\varepsilon}$. Das Kennzeichen des illativischen Infinitivs ist allgemein immer - $m s$, wo der Bindevokal geschwunden ist. ${ }^{1}$

Im folgenden untersuche ich die syntaktische Verwendung dieser beiden mordwinischen Infinitive, und zwar anf Grund des Materials, das in der von $\mathrm{H}$. PaAsones gesammelten und von PAavo RAviLA veröffentlichten Volksdichtung enthalten ist.

\section{Die Hauptregeln für die Verwendung des nominativischen Infinitivs}

1. Ein nominativisther Infinitiv wird i mmer dann verwendet, wenn das Prädi-

1 Zur Laut- und Formenlehre s. z.B. H. PaAsonen, Mordwinische chrestomathie S. 010; August Ahlqvist, Versuch einer mokscha-mordwinischen Grammatik S. 49; F. J. Wiedemann, Grammatik der ersamordwinischen Sprache S. 61; József Budenz, Moksa- és erza-mordvin nyelytan S. 90: PaAvo Ravila, FUF 20 S. 118; Enk k Itkonen, FI'F 29 s. 294 . 
kat ein intransitives, eine Bewegung ausdrückendes Verbum ist. Das $s u b j e k t d e s$ Prädikatsverbums dient a uch als Agens des Infinitivs, a $1 \mathrm{~s} \log \mathrm{isches}$ subjekt.

Beispiele, in denen Prädikatsverbum ein 'Kommen', 'Gehen', 'Aufbrechen', 'Laufen', 'Sichsetzen', 'Sichniederlassen', 'Sichbücken', 'Aufstehen', 'Haltmachen', 'Bleiben' ausdrückt:

kona sazo pšti uźeŕe di marto/ sẹ́eń koras mońeń kudoń tejerie 'dass die anderen mit scharfen Äxten kommen sollen, um für meinen Leib ein Haus zu machen' (E II 467) ${ }^{1}$; sado v́ dumso dumamo, v́e sov́ece kortamo, v́e suco sud'amo 'Kommt, um einhellig zu bedenken, um einhellig zu sprechen, um einhellig zu urteilen!' (E III 180); saka polanäj saka vasänäj kšida suskâma,/ kelmä vedńada pola.j matanäj a ton simemä 'Komm, Mann, komm, Gatte, Brot essen, frisches Wasser trinken, lieber Mann!' (M IV 87); niej sịnek tịnenk śukunamo 'Jetzt sind wir gekommen, um uns vor euch zu verneigen' (E III 139).

adla litova nalkseme, ad'a l'itova čaramo 'Komm spielen, Litova, komm tanzen, Litova!' (E I 286); a, vaj, ad'ado/ bojaravat jalğińeń/ ṕiśi bańaso paramo/ śveža v́ece valomo 'Kommt, Bojarinnen, meine Freundinnen, in der heissen Sauna zu baden, uns mit frischem Wasser zu übergiessen!' (E II 188); ad'a mońe·ń $u d o \cdot m o$ 'komm zu mir schlafen' (E III 278); ad’ä, täd'akaj, ošu, pazaru./ akša kalacaäń, täd'äj, ramama,/ šapama v́inań, täd'äj, śimamä! 'Komm zur Stadt, Mutter, zum Basar, um weisse Semmeln zu kaufen, Mutter, um bitteren Branntwein zu trinken, Mutter!' (M IV 108); ad’ada jalgat adada./ maźi tarań vanâma 'Kommt, Freundinnen, kommt die schöne Tara sehen!' (M IV 355).

aŕa·da śimə·mä jarca 'ma 'Kommt zu trinken, zu essen' (E II 522); ar'ta iga škajen śuduf ton äšelemä 'Komm, Iga, du Gottverfluchter, baden!' (M IV 130); aŕda praci samakâžu lädłamä 'kommt, Brüder, nach Samakush, um zı mähen' (M IV 436).

1 Die römische Ziffer bezeichnet den Band und die arabische die Seite des Werkes H. Paasonen - Paavo Ravila, Mordwinische Volksdichtung I-IV; $\quad \mathrm{E}=$ Ersamordwinisch, $\quad \mathrm{M}=$ Mokšamordwinisch; $\quad$ der Schrägstrich (/) bezeichnet die Strophen. 
a·taś a $\cdot j$ ataś, azo $\cdot \mathrm{ka} \cdot \mathrm{loń} k u n d a \cdot m o$ 'Alter, du, Alter, geh Fische fangen' (E III 273).

či-pas jakaś piśi bańaso paramo 'Der Sonnengott ging, in der heissen Sauna zu baden' (E II 471); tosa son ušịiźżä koda sịn jakaśt' patat jalakst anama 'Dann begann sie (zu erzählen), wie sie, Schwester und Bruder, auf Bettelei gingen' (E III 271-272); ved tị jakań sorôń jordama 'Ich ging um Kehricht auszuschütten' (M IV 14); ilä•t' jakań väärd'ä ṕeji nal'kâma 'Gestern Abend ging ich nach dem Oberdorf um zu spielen' (M IV 327).

l'ubava lisii luga lanks / mazi d'iga l'evks vanomo 'Ljubava geht auf die Wiese, um schöne Gänsekücken zu hüten' (E I 227); iśta liśnin kukov maro sporamo/ iśta jakin ćokov maro pelkstamo 'Also ging ich hinaus, um mit dem Kuckuck zu wetteifern, also ging ich (hinaus), um mit der Nachtigall $\mathrm{mm}$ die Wette zu singen' (E II 185); vana mon liśin valćḱińeń zoŕań vanomo 'Sieh, ich bin hinausgegangen, um das Morgenrot zu schauen' (E II 469).

koda ton molat stadań pańeme 'Wenn du die Herde austreiben gehst' (E I 46); mol's't gńedo-jeń kunda·mo 'Sie gingen den Braunen zu fangen' (E II 549); mol tam vel'e langa pŕań tramo, verin simme (ź-), lovažań śin treme, telań kośtamo 'Wir gehen in die Dörfer, um uns zu nähren, um Blut zu trinken, um Knochen zu zerbrechen, um Körper auszutrocknen' (E III 125); vaj molan mastôrôzen šarâma,/ mon narodôzen vardžama 'Ich gehe um auf meiner Erde umherzuwandern, um nach meinem Volk zu sehen' (M IV 13); mon af molan salama 'Ich gehe nicht stehlen' (M IV 427).

son tukšnoś najko stadań pańeme 'Najko ging die Herde austreiben' (E I 46); te śv́ečańt marto tukšnoś mastoroń krugom jutamo 'Mit diesem Licht machte er sich auf die Welt zu umwandern' (E II 472); koda tuit jakamo 'wenn sie zu gehen beginnen' (E III 130); esta songa tuś końafks jordama 'Dann ging auch sie einen Birkenkranz werfen' (M IV 17); eŕźäń ava par(o) ava/ tuś tišəń kajama 'Die Ersänin, die gute Frau, ging Heu vorwerfen' (M IV 426).

už kozoj kozoj sới te od torgovoj andomo 'wo, wo tritt der' junge Kaufmann ein um (die Pferde) zu füttern' (E I 165); 
kuśtima alov sịíe narv́ićka son sovaś/ vaj ṕižen [l. ṕizeñ] aškoń kuśtima alov purnamo 'die alte Bruthenne trat unter die Aussentreppe, um das Nest unter der Aussentreppe herzurichten' (E II 24); purla $k$ t jotašt, abe dama at suva-št' 'Burlaks gehen vorbei, sie treten nicht ein, um Mittag zu essen' (M IV 664); sâva·śt äšlemä 'Sie gingen sich zu baden' (M IV 881).

matŕań sajeme źińa sįrgakšnos' Sinja machte sich auf, Matrja zu holen' (E I 274); al'äźä sôr'ka·j əŕv́ä· ladama 'mein Vater wird sich aufmachen (dir) eine Frau zu freien' (M IV 69); ul'a ·ma

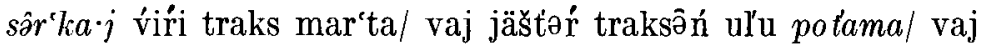
kaza vajəń ul'u pârda'ma 'Uljama geht in den Wald, wo Kühe sind, Geltkühe, Uljama, um zu melken, um Ziegenbutter, Uljama, zu sammeln' (M IV 231).

ćoraś čijs č čijs šabrav jovtamo 'Der Sohn lief und lief zum Nachbarn um zu erzählen' (E III 307).

jarcamo ozi stol'-ṕeńes ari 'Setzt es sich zum Essen, nimmt es am Tischende Platz' (E II 14); atäńt täjxt!̣́!̣ -za oza·ś ojm?ṣ́mäa: vä säl'mṭńt mańa ma 'Die Tochter des Alten setzte sich um sich auszuruhen und die Einäugige willfährig zı machen' (E III 244); ozak mar'tân abe-dama 'setze dich, um mit mir zu speisen' (M IV 829); ton molelet tâ ozalet vaksâzânza śarânkš́nemä 'du hättest gehen und dich neben ihn stellen sollen, um zu scheissen' (M IV 851).

koda sịn madñeśt v́eńeń udomo/ ašine činen a sịn učomo 'Als sie sich niederlegten, die Nacht zu schlafen, den hellen Tag zu erwarten' (E I 44); mad'neśt' v́eńeń udomo 'Sie legten sich hin, um die Nacht zu schlafen' (E II 138); son matś udâma salda'tś 'Der Soldat legte sich um zu schlafen' (M IV 827).

komakšnoś najko pillǵeń kaŕcéme 'Najko bückte sich um ihr Bein zu binden' (E I 46); odźoraś komaś k'inelelc símeme 'der Knabe beugte sich zur Kinelj nieder, um zu trinken' (E I 241); koma's' bolota's śimme' sovśe'm 'bückte sich ganz zu einem Sumpfe hernieder um zu trinken' (E III 282).

valk k'́eńč metamo, valk kŕeńč sętamo, valk puvamo, valk sel'geme 'Komm herunter, Rabe, um zu beschützen, komm herunter, Rabe, um zu behïten, komm herunter, um zu blasen, komm herunter, um zu spucken' (E III 86-87); vara-ka vall'ś $0 \cdot f$ to laṇks kaša do jarca·mo 'Die Krähe liess sich auf dem Bären nieder, um Brei zu fressen' (E III 281). 
stak ednä·zä, stak l'efkskäzä šuftôń kajama 'Steh auf, mein Kind, steh auf, mein Kind, um den Losungsstock zu werfen!' (M IV 126); śtaka śtaka aŕi·nuška śtava·jka/ načka śorôń, aŕi·nuškaj, s'tafñamä 'Steh auf, steh auf, Arinuschka, steh doch auf, um das feuchte Getreide, Arinuschka, (zum Trocknen) hoch zu legen' (M IV 338).

norožolks keṕeti mazị moroń moramo 'steigt die Lerche hoch, um ein schönes Lied zu singen' (E II 26).

lotkaśt ežneme 'da machten sie Halt um sich zu wärmen' (E III 314); ruzavaś lotkaś keṕṫín päskadma 'Die Russin blieb stehen, um den Korb zu füllen' (M IV 109); taza lotkan vajmama 'Hier mache ich halt um zu ruhen' (M IV 559).

koda kadovkšnoś najko ṕil'ǵeń kaŕćene 'Als sie blieb, um ihr Bein zu binden' (E I 46); uduma śkamịn kaduviin 'Ich blieh allein dort, um zu schlafen' (E III 293).

a atät sorats $i l a \cdot t s$ kud vanâma i kormań pidemä 'der Sohn des Alten aber blieb zurück, um das Haus zu überwachen und um Speise zu bereiten' (M IV 852).

Mitunter kann das Prädikatsverbum auch fehlen und der Infinitiv tritt allein auf:

»kudu, ednä·zä, nasi·lka,/ surôń pa·tšada jar'tsama,/ ravdža pozada simemä!»' '(Komm) heim, mein Kind, Nasilka, um Hirsepfannkuchen zu essen, schwarzes Dünnbier zu trinken!' (M IV 9).

Die einzige Ausnahme dieser 1. Hauptregel bildet das folgende Beispiel:

ad'ado míń ńej moldano,/ pokšunok (<-šon-) turtov ńej jovtams,/ ad'adoja mol'dano,/ satanailne jovtamo 'Kommt, lasst uns gehen unserem Oberhaupt erzählen, kommt, lasst uns gehen dem Satanail erzählen!' (E I 24).

2. Ein nominativischer Infinitiv wird i mmer dann verwendet, wenn das objekt eines transitiven Prädikatsverbums das Agens des Infinitivs, das logische subjekt ist. Das Objekt kann a l ch nicht bezeich net werden.

Beispiele, wo das Prädikatsverbum 'lassen', 'erlauben', 'verbieten', 'befehlen', 'cinladen', 'bitten', 'versammeln', 'bringen', 
'begleiten', 'vertreiben', 'werfen', 'schicken', 'setzen', 'geben', 'nehmen', 'erwarten', 'gebären', 'zurechtmachen', 'anspannen', 'wecken', 'Iehren', 'segnen', 'machen', 'sehen', 'hören' bedeutet:

noldat a noldat ton nujal' pokštaj udomo 'Lässt du (mich) ein, Grossvater Nujalj, zum Schlafen' (E I 166); noldasamak ton araś čopuda v́eń ṕečtamo/ noldasamak ton araś kuvaka v'eń ojmamo 'Lässt du mich ein, um die finstere Nacht zu verbringen, lässt du mich ein, um die lange Nacht hindurch zu ruhen?' (E II 240); no ldiñźe ala šanzo jarca·mo 'liess seine Pferde fressen' (E III 320); mon śiśom kizōt stada vanâma noldasa 'Ich sende ihn für sieben Jahre aus, Herden zu hüten' (MI IV 4); noldamaka ứa·šəฤkaj ton udâma 'Lass mich ein, Schwägoriı, zum Schlafen!' (M IV 329).

eźimim kadno bojar-avaks mon ěińem/ čopuda v́eń udomo 'Mein Bojarinnentum liess mich nicht die finstere Nacht schlifen' (E II 181); te kukovoś, tetakaj, eźinizé kadno čopuda ŕeń udomo/ te ćokovoś, tetakaj, ezinźet kadno kuvaka čiń ojmamo 'dieser Kuckuck, Vater, hat dich nicht die finstere Nacht über schlafen lassen, diese Nachtigall, Vater, hat dich den langen 'Tag über nicht ruhen lassen' (E II 247); akša f'edo siä $t$ kadj̇ź(ä) kuts aščama 'er liess die weisse Feda zu Hause sein' (M IV 155).

užo kevkśtasa mon v́eŕe-pazoń/ meri a meri kanstoń kandomo 'halt, ich frage Vere-pas, ob er es erlaubt eine Last zu tragen' (E I 32); pas merezę śv́eča laco valcto ṕinǵgen ṕeč́tamo, v́ejk'eń v́ejkeń iečkeme. 'Gott gewähre (ihnen), wie eine Kerze leuchtend das Leben zu verbringen, einander zu lieben!' (E II 160); to to jala·kskem, ei ń mere jaga baba·ń l'emd'e nolšta mo 'Da hast du es, mein Brüderlein, verbot ich dir nicht, vom Fett der alten Hexe zu lecken?' (E III 229); merí boja rote tolo-ńt mać 'a 'mo 'heisse deinen Bojaren das Licht auslöschen' (E III 321); totô t'śoraj izń märýaŕd’ä ot śätśśn k'iga jakama. 'Ich verbot ja, Junge, den Weg deines Onkels zu gehen' (M IV 812).

košarcamaí luvândâma 'mich zwingt er (auch) zu rechnen' (M IV 53).

polanzo terái a son jarsamo 'lud er seine Gattin zum Essen' (E I 254); ista teŕtinidiź ńin simeme jarcamo 'Wir haben dich so eingeladen, um zu trinken und zu essen' (E II 513); jaga

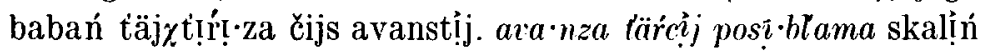


lńälga pogo•nc 'Die Tochter der Jaga-Alten lief zu ihrer Mutter und bat ihre Mutter bei der Verfolgung der Kuh zu helfen' (E III 247); al'äżä śéri jar'cama térni 'mein Vater ruft, er heisst (dich) essen kommen' (M IV 230).

potama anašt' 'sie bitten um das Melken' (M IV 416).

purnịtaja paro rodoń pokštat'neń/ sérnem koŕas kando-lazoń tejeme 'rufe die Grossväter des trefflichen Geschlechts zusammen, damit sie für meinen Leib einen Sarg machen' (E II 187); purnaíinek toń l'enitet kundamo 'wir haben uns versammelt um das Gedenken an dich zu feiern' (E III 15).

tuinkaja teta jurc/ teta jurtoń kirdeme/ ava jurtoń vanomo 'bringt ihn zur Wohnstätte des Vaters, damit er die Wohnstätte des Vaters besorge, damit er die Wohnstätte der Mutter betreue' (E II 231); ali ni tut kafta pilgen, ton, kirdemä,/ ali ni tut' nilä kilen, ton, juksema 'Oder hast du sie mitgebracht, damit sie dir die Beine halten würden, oder hast du sie mitgebracht, damit sie dir die vier Hosenschnüre lösen würden?' (M IV 504). v́l’əd'ä(ń) ušu ivań pârva-žaź| soń oćazôrti ivań služama v́äŕrd'ä škajəńd'i ivań truduma 'sie begleiteten Iva zum Dorfe hinaus, Iva, damit er dem Kaiser diene, Iva, damit er für Gott droben sich mühe' (M IV 360).

koda pańsịnze sịnst son śimeme/ kaŕks tapardamga (<-ŕd'-) a son ńej ṕesokka 'Als er sie zur Tränke treibt, (versinkt er) bis zu den Bastschuhschnuren in Sand' (E I 193); tetam panimim kudosto/ pele-v́eń škasto porasto/ ńeurosnoj škiníeste/ udi lomat puvtneme,/ mat'tań tolnet puvseme 'Mein Vater vertrieb mich von Hause, (sodass ich) zur Mitternachtszeit, zur unpassenden Zeit schlafende Leute wecken, erloschene Feuer anblasen (muss)' (E II 374); moŕa uśijas pańdadiź, ṕesokoń ṕikseń ponamo 'wir treiben euch auf eine Meeresinsel, damit ihr Sandseile dreht' (E III 172).

Ińejl'e śe suftamo tikšet'nen jorcįź okul'ća ušov 'Dann wirft man die zum Räuchern benutzten Kräuter aus der Einfriedung des Dorfes hinaus' (E III 168).

kučiźe ṕenǵeń baškir ava kéramo 'Die Baschkirin schickte ihn Holz zu hacken' (E I 202); ko•zo ku·čtan, a kučtan/ narmuška laco lifteme 'Wohin sollte ich dich schicken, gleich einem Vogel zu fliegen?' (E II 493); sirä skalịnnt alda pakšätńeń 
navo*zịn kịrga·ma kučcį・nizä '(Die Stiefmutter) schickte die Kinder den Mist unter der alten Kuh wegzuharken' (E III 237); ku t'sema sśt akl'akaj moń atän kudu/ tädänäzti penetsama jalât' śama 'Schickt mich, Schwägerin, nach dem Haus des Vaters, damit ich meiner Mutter klagen und jammern kann' (M IV 97).

źardo sto langozonzo pucaso jarcamo simeme 'wenn er ihn zum Essen [und Trinken] auf seinen Tisch stellt' (E III 207); pajarś putś kefnen vaks kafta avat jazama 'Der Bojar setzte zwei Frauen an die Steine, um zu mahlen' (M IV 889).

sai·źe béro mazịnde eje deśt i oza vtįndę marị nde užina jamo 'nahm das Kind auf die Arme und setzte sie (beide) neben sich, damit sie mit ihm ässen' (E III 268).

ofto-maŕnén'ksęn't orožijaś makssị lijańe pańeme 'Die Hundsrose gibt der Zauberer dann einem anderen zum Vertreiben (der Geister)' (E III 170).

už moń sajsamiź, koŕmakaj, ńekrutoks/ kuva kaḱińeń [1. kuvaka k'ińeń], avakaj, moleme,/ staka ružijań, koŕmakaj, kandomo 'werde ich als Rekrut genommen, Ernährerin, um lange Wege zu laufen, Mutter, um ein schweres Gewehr zu tragen, Ernährerin' (E II 81); sajiż te śripcikieńt moramo 'sie holten diesen Geiger zum Spielen' (E III 259); sävezä paťšada jar'tsama, / ravdža pozada simemä 'Sie nahm sie mit, damit sie Pfannkuchen esse (und) schwarzes Dünnbier trinke' (M IV 10); sävmak al'akaj sävmak teräjnäj trojtsän pazaru/ al'akaj tŕakaj krandazś vanâma/ akša paroťšań fata ramama 'Nimm mich mit, Vater, nimm mich mit, Ernährer nach dem Markt von Trojtskoje, Vater, Ernährer, um Karren anzusehen, um einen Schleier aus weissem, feinen Tuch zu kaufen!' (M IV 134).

mon učnija ḱel'ej kojmenze nej maro/ seŕńem koŕas sači modań čuvomo/ rungom koras b'iśor-modań čuvomo/ bojaravaks moń sẹininem valamo 'ich habe es mit seinem breiten Spaten erwartet, damit es für meinen Leib fruchtbare Erde aufgrabe, damit es für meinen Körper glasperlige Erde aufgrabe, damit es meinen Bojarinnenleib vergrabe' (E II 207).

šačftan kolma ćorańat,/ kaftônätńəń polatksâks, / kolmâćanä služa·ma 'Ich werde drei Söhne gebären, zwei von ihnen zu deiner Hilfe, den dritten zum Soldatendienst' (M IV 323); mez asusna pelaj svetkäti mon šatšftịnä,/ lama nuža·ń näimä lama 
valneń linlemä 'Was für ein Nutzen war darin, dass ich sie in die helle Welt gebar, viel Not zu sehen, viel (böse) Worte zu hören?' (M IV 600).

mez asuvâts pelaj svetkäti škaśt träst,,/ lama nuža·ń näjmä,/ lama valôń kulemä 'Was für ein Nutzen war dabei, dass ihr ihn in die helle Welt erzeugt habt, um viel Not zu sehen, um viel (böse) Worte zu hören' (M IV 591).

uriva·kstuma anôkstaf 'er ist fürs Heiraten zurechtgemacht' (M IV 356).

kildik ajgorośt' sokamo 'Spanne den Hengst an zum Pflügen' (E III 276).

mon avañä lnań mon acañälńań śtafcajnäa/mon lama traksâń mon lama traksôní potama 'Ich wecke die Frauen, die Frauen, um viele Kühe, viele Kühe zu melken' (M IV 355).

nän tonaftsịnä pervaj jar'tsama, sal'dä simemä 'es lehrt sie zuerst zu fressen, dann zu trinken' (M IV 362); ni nän tonaftsaź liemä 'und sie lehren sie fliegen' (M IV 431); tonaftâmajt tol matâma 'Du hast mich gelehrt das Fener zu löschen' (M IV 850).

ton baslavamak tiŕin avakaj torgovojks/ d'ošovinineste ton tetakaj ramamo/ už pitńejńeste o $\chi$ avakaj mijeme 'segne mich, liebe Mutter, zum Kaufmann, dass ich billig kaufe, Vater, dass ich teuer verkaufe, Mutter' (E I 164); baslacamak, tetakaj, čopuda v́eń udomo/baslavamak, koŕmakaj, bašḱir-v́ijd'e kekšeme 'Segne mich, Vater, dass ich die finstere Nacht über schlafen kann, segne mich, Ernährer, dass ich mich vor der Baschkirenhorde verstecken kann' (E II 238); baslavamak cańtamak/ ton viŕrezet sovamo/ viŕrga ukštorga jakamo/ v́íreń umaŕeń kočkamo 'segne uns für den Gang in deinen Wald, für das Streifen im Walde, für das Sammeln der Waldfrüchte' (E III 42-43).

cantese pas eramo/ cantesę pas aštomo/ i parodo nejeme/i mazịd'e nejeme/ śiśem ćorań tîramo/ śiśem urvań sajeme/ śiśem tejteŕeń maksomo 'Gott segne ihn, damit er lebe, Gott segne ihn, damit es ihm gut gehe, damit er Gutes sehe, damit er Schönes sehe, damit er sieben Söhne aufziehe, damit er sieben Schwiegertöchter nehme, damit er sieben Töchter (in die Ehe) gebe' (E II 213); čanifta·məśk ära·ma ašla·mä! 'Gebt mir euren Segen fürs Leben!' (E II 527); čañ tamak ton pakśazot/ moń 
umaŕeń kočkamo 'Segne mich (für den Weg) auf deine Feldmark, mich für das Sammeln der Früchte!' (E III 40).

čapakš praksḱe t'einek/ čapakš laco kasuma/ ṕenǵe laco rašlamo 'wir haben 'Teig angemacht, darnit er wie 'Teig hochgehe, damit er sich wie Holz vermehre' (E II 488); ton kšńi vasoloń tarka tujeźat, jakśtére tolco pultaźat, tejeźat pečkeme, tejeźat k'éramo 'Du, Eisen, bist von einem fernen Ort gebracht, du bist in rotem Feuer gebrannt, bist zum Schneiden gemacht worden, du bist zum Schlagen gemacht worden' (E III 134).

ašet näe-li stere-ma·r'ta sora jotama? 'Hast du nicht ein Mädchen mit einem jungen Mann hier vorbeigehen sehen?' (M IV 885); ašen näj stama lomat't' jotama 'aber solche Leute habe ich hier nicht vorbeigehen sehen' (M IV 886). ${ }^{1}$

marääzä avaŕdəma 'Sie hörte Weinen' (M IV 818). ${ }^{1}$

Die einzigen Ausnahmen von dieser Regel sind die folgenden Beispiele mit einem illativischen Infinitiv:

vaj a jomamga sịn śudịź/ vaj śiśem ijet seŕed'ḿe/ vaj śiśem godnẹt ńej pužmo./ vaj tarkińe maro vejkidñams/ vaj pralkske maro roonajams 'sie fluchten ihm nicht (gerade), dass er umkomme, (sondern) dass er sieben Jahre lang krank liege, dass er sieben Jahre lang leide, um einem Bette gleich zu werden, um einem Kopfkissen ähnlich zu werden' (E I 136-137); son śudįze śiśem iet' sered'ríeks/tarka marto - vejkid'nams 'Sie verfluchte ihn zu sieben Jahren Kranksein, dass er dem Bette gleich werde' (E II 535).

Die Hauptregeln für die Verwendung des illativischen Infinitivs

1. Der illativische Infinitiv wird immer dann verwendet, wenn das Subjekt

1 Im Ersamordwinischen wird in Verbindung mit Verben von der Bedeutung 'sehen' und 'hören' das Partizip oder der Ablativ des Infinitivs verwendet: vaj ki ńeiże torgaš avaŕd'i/ už k'i ried'iże torgaš melafti 'Wer sah den Kaufmann weinen, wer bemerkte, dass der Kaufmann traurig war?' (E II 84); kona marasi zora porańe son mori 'Wer hört sie in der Morgenrotstunde singen?' (E II 26); vaj k'i nieiże avaŕd'émed'e? 'Wer sah ihn weinen?' (E II 17); uk k'i ńeiże melavtumado 'Wer sah ihn traurig sein?' (E II 98).

7 - Finnlsch-ugrische Forschungen 
eines intransitiven Prädikatsverbums das logische Objekt oder das Adverbiale d e s Infin it i vs ist (d.h. dann, wenn das Subjekt des Satzes Ziel oder Ort, Mittel udgl. des im Infinitiv enthaltenen Handelns ausdrückt). Ein eventuelles Agens des Infinitivs wird durch den Allativ bezeichnet.

Beispiele, in denen das Prädikatsverbum ein 'Geraten', 'Taugen', 'Wachsen', 'Haben' (gewöhnlich negativ) oder 'Sein' (gewöhnlich negativ) ausdrückt:

tońet čavoms ravž ańd'amo a pongan 'Ich werde schwarzbrauner Andjamo, von dir nicht getötet werden' (E I 373).

moń kšinä• uli täd'akaj jar'cams af moli/ pojlańä ul'i täd'akaj simams af moli 'Brot habe ich, Mutter, aber es geht nicht zu essen, Trank habe ich, Mutter, aber es geht nicht zu trinken' (II IV 307).

fśak simi jar'tsaj, šra lanks putâms af pečkä̈j 'Jeder trinkt und isst es, (aber) es taugt nicht dazu, auf den Tisch gelegt zu werden' (M IV 619); kitškôr, muvôr nal'kš, kädezt sävems af petšk $\ddot{a} \cdot j$ 'Ein schiefes, krummes Spielzeug, in die Hand zu nehmen taugt es dir nicht' (M IV 632); l’äjś ponań, pred'ńək'ś pa-

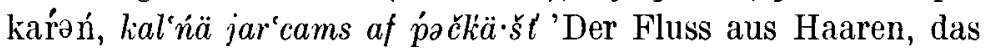
Zugnetz aus Knochen, die Fische nicht essbar' (M IV 641).

ša čozo osksońt oznoms šači śurost 'möge ihr wachsendes Getreide zum Opfern des Opfers wachsen' (E III 32).

el'i araś jarcams moń ǩšinem/ už el'i araś killdems rakšiniem 'Habe ich nicht Brot zu essen, habe ich nicht ein Pferd anzuspannen?' (E I 145-146); araś nujems, tejter' taka, tarvazom' Ich habe keine Sichel um zu schneiden, Mädchen' (E I 439); mazị ulańa moń tońt urvakstoms moń uli dobram 'schöne Uljanja, ich habe Eigentum, um dich zu verheiraten' (E I 475); uks araś níinek kildems išmemek 'wir haben kein Pferd anzuspannen' (E I 487); koli aras [l. araś]/ ninińek kaźems jarmakot 'Wenn du nicht Geld hast, uns zu beschenken' (E II 236); araś, tetkaj, mońe arams tarkinel aras', koŕmakaj, mone ojmams ugolne 'Es gibt, Vater, keinen Platz für mich zum Hintreten, es gibt, Ernährer, keinen Winkel für mich zum Ausruhen' (E II 238); langə̃zt ščams aš

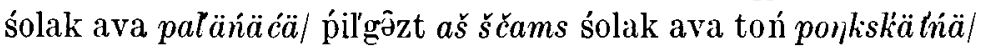

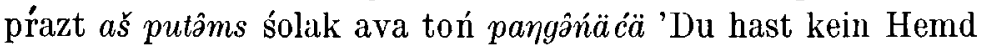


dich zu kleiden, ungeschickte Frau, du hast keine Hosen deine Beine zu kleiden, ungeschickte Frau, du hast keine Haube auf deinen Kopf zu setzen, ungeschickte Frau' (M IV 64-65); al'i jar'cams vaśäj polaj korma aš/ al'i śiməms vaśäj polaj pojla aš 'Gibt es nicht, Gattin, Speise zu essen, gibt es nicht, Gattin, 'Trank zu trinken' (M IV 94); kud uštâms ajaš toń ṕeıǵariätnää 'du hast kein Holz zum Heizen des Hauses' (M IV 200); ali jar'cams aš tama id'ńä·źä kšsinä ćä 'Hast du nicht, Tama, mein

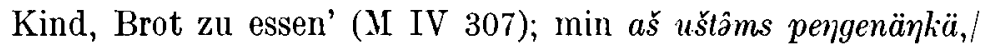
min aš tälams śorânäjjkä 'Wir haben kein Holz zum Heizen, wir haben kein Getreide zum Dreschen' (M IV 602).

Wenn das Prädikat ein Verbum mit der Bedeutung 'es gibt', 'haben' oder ein blosses Verneinungswort ist, kann das Subjekt anch unbezeichnet bleiben, nur das Relativpronomen weist darauf hin. Das Objekt- und insonderheit das Adverbialverhältnis zwischen Infinitiv und Subjekt wird durch die Flexion des Pronomens deutlicher. Die Konstruktion entspricht den finnischen Ausdrücken ei ole missä tehdä 'es gibt nicht wo tun', d.h. 'es ist kein Platz da, wo man es tun könnte', ei ole millä tehdä 'es gibt nicht womit tun', ei ole mitä tehdä 'es gibt nicht was tun' usw. Beispiele:

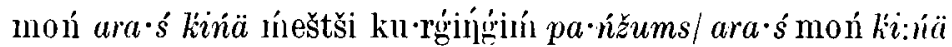

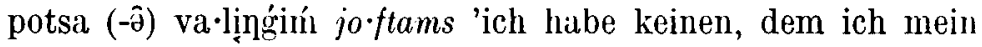
Herz öffnen könnte, ich habe keinen, dem ich das Wort in meinem Innersten sagen könnte' (E II 436); maksodo šači śuro, sovíi eíne, ulezę miejsę l'eńenk kundams 'Gebt wachsendes Getreide, einkommendes Vermögen, möge etwas dasein, womit euer Gedächtnis begangen werden kann!' (E III 4); tejteŕne a koda i kortams 'Es war dem Mädchen (einfach) nicht möglich, zu sprechen' (E III 338); i targasa, i täl'asa, šra lạk's putâms aš mezä 'Ich rupfe es aus und dresche es, (und doch) kann man daraus nichts auf den Tisch legen' (M IV 619); aš koda jotams təinza 'Sie kann auf keine Weise hinüberkommen'

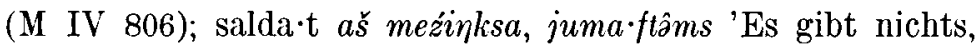
weswegen man den Soldaten umbringen könnte' (M IV 824); jotams aš mezsa (< meźsa) 'Es gibt nichts, womit man hiniuberfahren kann' (M IV 831). 
2. 1) r illativische Infinitiv wird immer dann arebraucht, wenn das objekt des transitiven Prädikatsverbums das logische Objekt oder Adverbiale des Inf i n it ivs is t. Das Agens des Infinitivs wird durch den Allativ ausgedrückt.

Beispiele, in denen das Prädikatsverbum 'lassen', 'befehlen', 'fordern', 'bringen', 'führen', 'werfen', 'geben', 'nehmen', 'suchen', 'finden', 'ansetzen', 'umwerfen', 'drehen', 'machen' oder 'in Ordnung bringen' bedeutet:

ili kado moń ejedeń e(j)kakson śirotaks/ śeźganne varakane salśems (od. salćems) 'lasse meine Kinder, meine Jungen, nicht Waisen werden, sodass sie von Elstern und Krähen geraubt werden' (E II 544); kudịnğiti kadįz ślirit' kujti śäuvums 'In dieses Häuschen liessen sie das Mädchen, damit der Lindwurm sie fresse' (E III 219); numsta paśl'e-dnaj uma ț andôms tarvasnəńd'i jar'cams rośt kadândiุšt', śorôśs šačəza 'Wenn man auf dem letzten Ackerbeet erntet, lässt man, um sie zu füttern, den Sicheln Roggen zum Fressen übrig, damit das Getreide wachse' (M IV 719); lazksńəńd'i valmat kadândị̌t', kulâfńəńd'i vanândâms 'In den Särgen lässt man Fenster zurück, damit die Toten hinausschauen können' (M IV 725).

eś sel v́eden dị noldịńl eś seriním elšelems 'ich habe meine eigenen Tränen strömen lassen, damit mein eigener Leib darin bade' (E II 454).

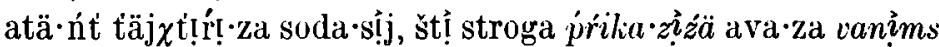
'Die Tochter des Alten wusste, dass ihre Mutter ihr streng befohlen hatte Acht zu geben' (E III 244).

kažnaj šinä· kujs antsel lomat't' sivâms 'Jeden Tag forderte der Lindwurm Menschen, um sie aufzufressen' (M IV 870).

äṕä·t tujsįź ombitśä čista śtir't kujti sáäums 'Am folgenden Tage brachten sie wieder die Tochter, damit der Lindwurm sie fresse' (E III 220).

kolmițśid'ä äpä̈t $i$ ' liź kujti śävums 'Sie brachten sie ein drittes Mal wieder, damit der Lindwurm sie fresse' (E III 220).

kajäś tśitúäŕl’k' peštä sịnst andums 'warf ihnen ein Tschetwerik Nüsse zum Essen hin' (E III 233).

maksịk soždịnet parịnet eŕra tarkas sịgamsto, érva tevnes 
kundamsto šoždịńestẹ kundams, parịnneste tejems 'Gib dein Leichtes und Gutes, wohin wir auch gehen, gib, dass wir leicht anfassen und gut machen können, was für eine Arbeit wir auch anfassen!' (E III 18-19); maksizz surįigist suskums 'Da reichten sie ihren Finger zum Beissen hin' (E III 233); maksíżä śiśtm säl'ńı̈ pranza čavśı́ms 'Die Siebenäugige reichte den Kopf hin zum Lausen' (E III 238); atinǵää maksịzäa pranza ćavśums '(Da kam) ein Alter (und) reichte seinen Kopf hin zum Lausen' (E III 273).

tosa ńi sääijä juva•nń alošat pŕa(n)ts iešsams 'dann habe ich .Juvans Aljoscha (zu mir) genommen, um ihm den Kopf zu lausen' (M IV 190).

'iešnit' eżi i', kormakaj, mońe k'ekšems ugolne 'hast du, Ernährer, einen Winkel für mich gesucht als Versteck' (E II 238); sịn iäšiz' kunda'ms sonza 'Sie versuchten ihn zu fangen' (E III 265).

sịn śek!ń vanịk koda bị aj alašat aj lija mezìt salams 'Man muss jeden Augenblick fürchten, dass sie Pferde oder anderes zil stehlen suchen' (E III 293).

eź mujt ramams son pala 'aber er fand kein Hemd zum Kaufen' (E I 508); son af muj vastânä iärâzams 'Sie fand keinen (passenden) Platz, um zu zickeln' (M IV 802).

siiča·s ozaftâżä śimdems andâms 'Sogleich liess sie ihn sich setzen, um ihm Trinken und Essen zu geben' (M IV 826).

koda toń tätat moń prafca·mam pačkı̣ms i k'eŕča samam 'wenn dein Vater mich zum Schlachten umwirft und mich bindet' (E III 246).

kadịk' i sịn piks ponît' skalịnt kerécams 'Nun sollen sie auch den Strick drehen, um die Kuh festzubinden' (E III 245).

fijeda veńškä jotams 'Macht ein Boot zum Überfahren' (M IV 833).

la tśak morkš i sedaft ozśams 'Bringe einen Tisch in Ordnung und Bretter um zu sitzen' (M IV 832).

Das Objekt des Prädikatsverbums kann manchmal auch unbezeichnet bleiben, wenn keine Unklarheit entsteht:

vaj purnaś serńnas sonze polazo son jarsams nej 'Seine Gattin richtete (alles) zum Essen' (E I 59); maksan simems 'ich gebe ihm zu trinken' (M IV 630) (vgl. fi. antaa syödä, juoda); kadâdä 
modinä nilä vajmen tale·kańat,/ kosta säran teest tat'śkanä kajams 'Ihr habt mir die (Steuer)anteile von vier Köpfen überlassen, woher werde ich (Geld) nehmen, um ihre Stewern zn bezahlen?' ( M IV 600).

Die beiden folgenden Beispiele sind Ausnahmen von dieser 2. Regel:

i kaftoso son cotil tejter takań lazeme/ bojar-avań locomo 'ja, Zwei (hunderte) rechnet er hin, um Mädchen zu beschenken, um Bojarinnen zu bescheren' (E II 236); lamo jarmak sajertit' tejteŕ-takań kazeme,/ bojar-avań lovomo '(dann) hättest du viel Geld bekommen, um Mädchen zu beschenken, um Bojarinnen zu bescheren' (E II 237).

3. Der illativische Infinitiv wird allgemein immer dann gebraucht, wenn der Infinitiv die Bestimmung eines Nomens (eines abstrakten Substantirs oder Adjektivs) is t.

Beispiele für die Konstruktion 'Lust (haben), Leid, Schande, zuwider, schwer, gut, ungerecht (sein), etwas zu tun':

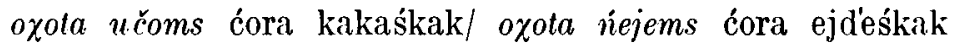
'(aber) er hatte auch I,ust den Sohn zu erwarten, (aber) er hatte auch Lust das Kind zu sehen' (E I 397); zal istoźams burojeze/ zal jomaftoms burojeze 'Es tat ihm leid, seinen Brannen zu töten, es tat ihm leid, seinen Braunen umzubringen' (E I 397); vizks ušu liśams/ prati vn(a) jalg(a) jotks liśams 'Es ist eine Schande hinauszugehen, es ist (mir) zuwider unter die Freundinnen zu gehen' (M IV 235); tonätka·k aštụms vakscịn!̣ skušna 'Es wird dir ja auch langweilig, bei uns zu sein' ( F III 244); pl'əmä·ńnəkâniäj, t’udna satâms 'es ist schwer es zu schaffen' (M IV 814); vaj erams pokštomo a ńej a paro 'Ohne Oberhaupt zu leben ist nicht gut' (E I 91); fkäti maksâms abi·dna 'Es einem zu geben wäre ungerecht' (M IV 874).

Ausnahme: líinenék' pora tujeme '(So) ist es (auch) für uns Zeit fortzugehen' (E II 262); pora teinek'/ ombo v́el'ej ardomo 'Es ist Zeit für uns jetzt ins andere Dorf zu fahren' (E II 316).

Beispiele, in denen der Infinitiv ein als Prädikativum stehendes Adjektiv bestimmt und in denen das Subjekt des Satzes das logische Objekt oder Adverbiale des Infinitivs ist (Kon- 
struktionen, wo das Subjekt das logische Subjekt des Infinitivs wäre, sind mir nicht begegnet):

uk paro paro sokams modazo/ śed'ejak paro strojams ( $<$ sro-) iirnezę 'Gut, gut ist ihre Erde zum Pflügen, noch besser ist ihr Wald zum Bauen' (E I 150); sịn at maštuíit' jar'tsams 'Sie taugten nicht zum Essen' (E III 231); simems kelmenä '(Sein Wasser) ist kalt zum Trinken' (M IV 87); selmeträ ožnat targams možnat 'Deine Augen sind wie Glasperlen, man kann sie herausziehen' (M IV 523); kalś jar' cams para tô śova-dônza lama 'Der' Fisch ist gut zu essen, aber er hat viel Gräten' (M IV 695).

\section{Ergänzungen und Śchlussfolgerungen.}

Im Lichte der oben dargestellten Hanptregeln scheint die, Verwendung der Infinitive im Mordwinischen ganz klar umrissen zul sein: ein nominativischer Infinitiv wird immer dann verwendet, wenn das zur Konstruktion gehörende, inhaltlich zentralste Nomen, das Subjekt oder Objekt des Prädikatsverbums das Agens des durch den Infinitiv ausgedrückten Handelns ist; in allen anderen Fällen wird der illativische Infinitiv benutzt. ${ }^{1}$ Diese Feststellung trifft jedoch nicht bei solchen nominalobjektlosen Verben zu, die keine Bewegung ausdrücken und deren Subjekt gleichzeitig das logische Subjekt des mit ihnen verbundenen Infinitivs ist. In einigen Fällen kann nämlich dann in Ergänzung der 1. Hauptregel ein illativischer Infinitiv stehen. Die verschiedenen Verben werden unterschiedlich konstruiert. Zuerst seien Beispiele für Verben angeführt, mit denen nach meinen Belegen nur ein illativischer Infinitiv verbunden wird und die 'ertragen, aushalten', 'sprechen, übereinkommen' und 'drohen' bedeuten:

1 Dieser Regel folgen auch einige Infinitivbeispiele in den Gesamtdarstellungen verschiedener Ersadialekte in dem Werk Oчерки мордовских диалектов (Том 1, Саранск 1961), S. 56-59 und 263. Genau die gleiche Zweiteilung lässt sich für den Gebrauch des 1. Infinitivs und des Illativs des 3 . Infinitivs im Ostseefinnischen vollziehen. S. Pauli Saukkonen, Itämerensuomalaisten kielten 1. infinitiivin ja 3. infinitiivin illatiivin kantasuomalaisista funktioista, Manuskript in der Wörterbuchstiftung (Sanakirjasäätiö), z.B. S. 266. 
iščo vanumak kafto jaḱi ṕilǵgeva/ kafto tei ked'ńeva/ cidardịt' araś v́e čiń robotank robotams 'Sieh auch meine zwei schreitenden Beine, meine zwei arbeitenden Hände, ob sie die Arbeit eines Tages bei euch ertragen' (E II 259).

salda·t śekiń kor'tă veńtśams '(Aber) der Soldat wollte immer noch getraut werden' (E III 223); sinn nejníiśt i kor'tas't ứva·ks saźums śtiŕt' 'hatten sie sich gesehen und waren übereingekommen sich zu heiraten' (E III 264).

piksâms šukšta·nza 'er droht dich zu prügeln' (M IV 119); šuk šândâśt' šavâms 'man hat ihn umzubringen gedroht' (M IV 894).

Beispiele, wo ein Verb mit der Bedentung 'wollen', 'denken', 'wagen', 'taugen', 'reichen', 'Zeithaben' oder 'sich fertig machen' entweder mit einem nominativischen oder mit einem illativischen Infinitiv verbunden werden kann:

ńiń koteli ńej estenze jarsamo 'Wir wollten von ihm fressen'

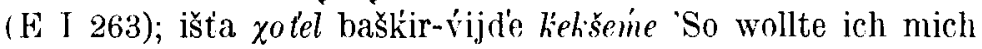
vor der Baschkirenhorde verstecken' (E II 249); zo čt ponosoń a son sajeme/ vaj staka kanstoń a son kandomo 'will sich eine Bürde holen, (will) eine schwere Last tragen' (E I 32); vaj ločot son ṕizeń aškoń purnamo,/ vaj kočot son mazi l'evkskeń Tivteme 'Er will ein Nost machen, er will schöne Junge aus-

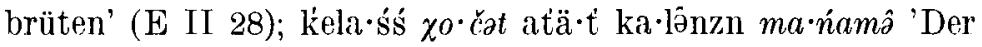
Fuchs wollte den Alten um seine Fische betrügen' (M IV 836).

ridna nadijat teśkende par'śte éamo 'du hoffst wohl (dort) hesser zu leben als hier' (E II 109).

a son a ve čkil odo-v kievkśne mue aber er liebte es nicht nochmals zu fragen' (E III 319).

kolmôtsä kezônä· piksa·jeń tạ́uś šacâms jorasị 'im dritten Jahre will Piksajs Tarju (ihren Mann) töten' (M IV 86); a k'elaźśs joraj atät kalnzôn kängalams 'Der Fuchs aber wollte den Alten um die Fische betrügen' (M IV 839); a kelazś joraj atät kalōnzân vaśkaftems 'Der Fuchs wollte dem Alten die Fische. stehlen' ( $\mathrm{X}$ IV 843).

iśta aŕcin paniemef 'so habe ich gemeint, dich zu verjagen' (E III 197).

ńiške-pas maro norov-pas/ dumaśl ućaskań jacšeme 'Nischkepis und Norov-pas dachten Glück zu spenden' (E I 64 ); v́idna 
dumide putoń kudosto javomon 'ihr habt wohl gedacht, mich aus dem gebauten Hause zu entfernen' (E II 165); duma 's $t^{\prime}$ stada·ń saje·me/ sịn tabu.noń pańe·me'dachten die Herde zu nehmen, die Herde fortzutreiben' (E II 549).

službasa služams duma·dnaś/ pokš vaśiń śtíriza '(Daher) gedachte im Kriegsdienste ihre grösste, erste Tochter zu dienen' (E II 112); sịn duma dnas't' sonza jumaftums 'da gedachten sie ihn umzubringen' (E III 263); äśtä·ń org? d’ums duma·dnakšnịn 'Ich dachte zu fliehen' (E III 295).

apak śreča a śmejan putoń kudozonk sovamo 'wage ich nicht, ohne dass man mich empfängt, in euer gebautes Haus einzutreten' (E II 204).

polaftâzä di af smedndaj kizefiems 'er hat ihn getauscht und wagt nicht zu bitten' (M IV 831).

son udalakšnoś bogdaške kuṕećk'eks/ śiśem vel'eva bogdaške jakamo/ śiśem tị̌sićań bogdaške sajemie/ son d'ošovasto bogdašk'e ramamo/ už pitńejńeste bogdašk'e mijeme 'Bogdaschke taugte gut zum Handelsmann, sieben Dörfer zu besuchen, siehentausend zu bekommen, billig zu kaufen, teuer zu verkaufen' (E I 168); son eś udala savań téruša sokamo 'Savas Terjuscha taugte nicht zum Pflügen' (E I 383).

šamat'sen vel'tams toń końdästeza 'Möge er zum Verhüllen deines Gesichtes taugen!' (M IV 266); šumbra- šińa·sa kańnems lońde·steza 'sei es dazu geeignet, um es in Gesundheit zu tragen' ( M IV 545).

vaśńa vanumak alka rošta sẹ́ńeva/ satị a satị eźem prat kuvalt v́eńemme/ eźem pŕat kuvalt v́eńemme ašo kocso v́el t'avmo 'schaue zuerst meinen Leib, der einem niedrigen Haine gleicht, ob er genügt, sich auf deine Bank zu strecken, sich auf deine Bank zu strecken, sich mit weissem Linnen zu decken' (E II 258).

son rakšań kirdems vijze eś sato 'seine Kraft reichte nicht hin, das Pferd zu halten' (E I 240); tińenkkak satoc moń sé̛ińem melavtoms/ tịnenkkak satoć moń rungưińem melavtoms auch euch hat mein Leib Sorge gebracht, auch euch hat mein Körper Sorge gebracht' (E II 457); vajğeleś satozo eś v́iška segrem kovulams/ raluẹtíe satost bojar-avaks-činem avarićtems/ ol'aks-čińem kukorctoms 'Die Stimme soll hinreichen, über mei- 
nen kleinen Leib zu trauern, die Worte sollen hinreichen, mein Bojarinnentum zu beweinen, über meine Freiheit zu klagen wie ein Kuckuck!' (E II 468); koda mašśt sịń v́ijd'e valdo, a satị jakams moćest i maźalost 'Wie sie ihre Kraft verloren haben, wie ihre Kraft und Stärke zum Gehen nicht ausreicht' (E III 130); vancamań prasta šäjärǵää/ sati əli moń šäjärnáäźä/ kääd'ənc ṕerfkä tapardams 'er betrachtet mein Haar auf dem Kopfe, ob mein Haar hinreicht, um (es) um seine Hand zu wickeln' (M IV 462).

eś kener't maksmo baslovka '(Aber) er hatte nicht (mehr) Zeit den Segen zu geben' (E I 295); ezín kener't kolmo čińet eram. 'Ich habe nicht einmal drei Tage zugebracht' (E II 292); a·ńćak kenér't ponkso't nolda 'mo 'Sei nur bereit, deine Hosen zu öffnen' (E III 317).

mon äzin kändir ma tums fanaŕt 'Ich hatte kaum Zeit die Laiterne auszulöschen' (E III 298); son bjednịjś äź kändir i a $\chi$ joftams 'Die Arme hatte nicht einmal Zeit "Au!" zu sagen' (E III 300); ańtśak kenerśt nä śudufnä panäti suva $m s$ melganza laśkś sirä pinä igań aläńats 'Kaum waren die Armen in die Sauna eingetreten, lief hinter ihnen her Igas Vater, der alte Hund' (M IV 130); son eź kener kädents veneptems, kanttnä keneŕsí kaladâms 'Er konnte noch nicht die Hand reichen, als die Baumstämme schon herabrollten' (M IV 868).

už lomat purnįt' sokamo/ vaj lomat purnįt izamo 'machen sich die Leute fertig, zu pflügen, machen sich die Leute fertig, zu eggen' (E I 227).

davajka purnak ton mazị polam sazorośt andoms ńej 'Wohlan, mache dich bereit, meine schöne Gattin, die Schwester zu speisen!' (E I 59).

udâk kuva·t' id'a·kańäj kast kuràk oćusta/oću uma·ń sokama/ lama śorôń vidəma/ ŕetä lạksa uskâma/ oću kapań vačkama 'schlaf lange, Kindlein, wachse schnell gross, um grosse Ackerstücke zu pflügen, ım riel Getreide zı säen, um (es) mit fünf (Pferden) einzufahren, um grosse Schober zu häufen' (M IV 319-320); kə̂va·t udị, kurâk kasị,/ kvaka uma·ń sokama,/ keli lexań lazâma 'schlafe lange, wachse schnell heran, um lange Ackerstücke zu pflügen, um breite Streifen Ackerland zu furchen' (M IV 413). 
Beispiele für Verben mit der Bedeutung 'beginnen', 'bitten' und 'können'; sie werden nur mit einem nominativischen Infinitiv konstruiert:

narod karmi araídeme 'Das Volk beginnt zu weinen' (E I 11); vaj rana karmaś kodamo 'Frühzeitig begann sie zu weben' (E II 67); karmaśt' śe orozońt joŕtnéme 'sie begannen den Haufen (in alle Winde) zu werfen' (E III 77); śor avaś larmaś ṕejođəmânza '(Da) begann die Getreide-Mutter sie auszulachen' (II IV 19); vaj esta karmaś jasna kor'tama 'Ach, da begann er klar zu sprechen' (M IV 275).

śeste praś il'uša sered'me 'Da begann Iljuscha zu erkranken' (E II 535).

i ṕedaś śŕipkaś lomańks moramo 'Und die Geige begann wie ein Mensch zu singen' (E III 259).

už kozoj kozoj kevkśti te od torgovoj udomo 'Wo fragt der junge Kaufmann $1 \mathrm{~m}$ Schlaf(statt)' (E I 165); i kevs val'ma loj udo'mo' Und sie bat (hineinkommen zu dürfen um) unter einem Fenster zu schlafen' (E III 266); kevkśtaś udomo 'Er fragte, um zu schlafen' (E III 324).

a maštan v́ed'eń, avaj, kandomo,/ a maštan ṕenǵeń, avaj, kandomo 'ich bin nicht imstande Wasser zu holen, Mutter, ich bin nicht imstande Holz zu holen, Mutter' (E I 196); sonid'ä.

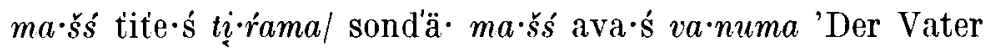
vermochte sie zu erziehen, die Mutter vermochte sie zu betreuen' (E II 420); možot ńiń a mašstana oznâma, enialdâma 'Vielleicht vermögen wir nicht z11 beten, zu flehen' (E III 186); karu af maštị kšstirdamä kodama '(Aber) die Fliege kann nicht spinnen und weben' (M IV 63); čofkśś kosônga ašəź tonafńä, a morama maštį 'Die Nachtigall hat man nirgends gelehrt, aber singen kann sie' (M IV 706). ${ }^{1}$

Das Prädikatsverbum kann in diesen Fällen - ebenso wie ein eine Bewegung ausdrückendes Verb - mitunter unbezeichnet bleiben:

d'ijakon sed'e kurok sokamo 'Der Diakon begann eilig zu pflügen' (E III 309); tatar sudamo martondo 'Der Tatar wollte (deswegen) einen Prozess mit ihm machen' (E III 331).

1 Anders im Ersadialekt von Prialatyrsk nach F. P. Markov: A maštan korklems 'Я не умею говорить' (Очерки мордовских диалектов S. 57). 
Man müsste mehr volkssprachliches Material haben, um schlussfolgern zu können, nach welchem System die Infinitive bei solchen Verben verwendet werden. Diese Frage ist eng mit dem Problem der Entstehung der Infinitive, besonders des nominativischen Infinitivs verknüpft. Der illativische Infinitiv entspricht den Erwartungen und erklärt sich auf dieselbe Weise adverbiell wie die lativischen Infinitive mancher anderer Sprachen, z.B. der ostseefinnisch-lappische $k$-lativische 1 . Infinitiv und der ostseefinnische Illativ des 3. Infinitivs oder der vermutlich lativische Infinitiv der permischen Sprachen auf $-n i(s),-n \hat{\imath} .^{1}$ Wenn jedoch der nominativische Infinitiv ursprünglich der Nominativ eines Verbalnomens ist, muss er als Bestimmung des Verbs das Objekt gewesen sein. Der Nominativ kann im Mordwinischen der Objektskasus sein — zum Ausdruck des Indefiniten $^{2}-$, und somit ist die Erklärung möglich, dass die ranze Verwendung des nominativischen Infinitivs als Objekt einiger transitiver Verben begonnen hat, solcher Verben wie z.B. die oben angeführten, gerade den nominativischen Infinitiv verlangenden Zeitwörter mit der Bedeutung 'beginnen', 'bitten' und 'können'. In einem Zusammenhang folgender Art ist z.B. die Entwicklung vom Nomen zum Infinitiv durchaus möglich: koda karmatank míń, polańakaj, éamo 'Wje beginnen wir, Gattin, (weiter) zu leben?' (E I 386). Jene Verben wiederum, die nach den oben zitierten Beispielen mit dem illativischen Infinitiv konstruiert werden, sind erwartungsgemäss meist intransitiv oder erhalten eine lativische adverbiale Bestimmung. Diese Zweiteilung im Gebrauch der Infinitive ist jedoch nicht kategorisch; die Beziehungen können sich später vermischt haben. $\mathrm{Zu}$ einer endgültigen Klarheit wird man erst mittels der Etymologien der Prädikatsverben kommen.

Das schwierigste Hindernis für die Annahme, dass der nominativische Infinitiv auch ursprünglich ein Nominativ ist, liegt gerade in dem reichlichen heutigen Gebrauch des Infinitivs nach den zwei von mir aufgestellten Hauptregeln. In den $\mathrm{Zu}-$ sammenhang von Verben, die eine Bewegung ausdrücken, passte

1 Günter Stipa, Funktionen der Nominalformen des Verbs in den permischen Sprachen, MSFOu 121 S. 60.

2 F. J. Wiede Man n, Grammatik der ersa-mordwinischen Sprache S. 45. 
der Nominativ gar nicht; ebenso wenig eignete er sich als Objekt eines solchen transitjven Verbs, das bereits ein (als logisches Subjekt des Infinitivs fungierendes) Nominalobjekt besitzt. Die einzigen in Frage kommenden Konstruktionen wären die nominalobjektlosen 'eine Handlung erlauben, verbieten, erbitten, erwarten, segnen, sehen, hören'. Es ist theoretisch möglich, dass sich die Entwicklung vom Nomen zum Infinitiv durch einen folgenden Gebrauch hätte vollziehen können: uči avanzo kuluma/ uči firanzo jumamo 'Sie wartet auf den Tod ihrer Mutter, sie wartet auf das Sterben ihrer Ernährerin (E I 142). Dann wäre eine Verschiebung der syntaktischen Gliederung vor sich gegangen: 'sie erwartet das Sterben ihrer Mutter' > 'sie erwartet ihre Mutter zu sterben'. Da auch der Genitiv als Objektskasus auftritt - zum Ausdruck des Definiten ${ }^{1}$ - kann das Genitivattribut des Infinitivobjekts gut zum direkten Objekt des Prädikatsverbums geworden sein: 'das Handeln jemandes segnen, erbitten, erwarten' > 'jemanden segnen, bitten, erwarten zu tun'. Diese Vermutung wird speziell durch den Umstand gestützt, dass im Zusammenhang mit dem Infinitiv das Objekt ohne Ausnahme i $m$ m er, auch wenn es indefinit ist, im Genitiv steht, wie bereits Wiedemann anführt: karmaź jovksoń jovtamo 'er fing an eine Geschichte zu erzählen (die Erzählung einer Geschichte)'. ${ }^{1}$ Damit aber eine derartige Entwicklung zum Gebrauch des heutigen nominativischen Infinitivs hat führen können, müssen die folgenden Voraussetzungen bestanden haben: Jene gewöhnlichen Konstruktionen mit einem nominativischen Infinitiv wie z.B. 'jemanden bringen, setzen, geben, nehmen zu tun' sind erst entstanden, als in den Konstruktionen 'eine Handlung jemandes segnen, erbitten, erwarten' bereits die heute geltende Verschiebung der Gliederung stattgefunden hatte: 'jemanden segnen, bitten, erwarten zu tun'. Der nominativische Infinitiv hat speziell die Bedeutung der aktivischen Handlung - als Gegensatz zur passivischen Funktion des illativischen Infinitivs - erhalten und ist nur für jene Fälle allgemein geworden, in denen Subjekt oder Objekt des Prädikatsverbums das Iogische Subjekt des

1 WiedemanN, a.d.O. S. 45. 
Infinitivs bilden, so dass er einerseits auch mit Verben verbunden werden konnte, die eine Bewegung ausdrücken, sich andererseits aber z.B. nicht in Konstruktionen wie 'die Verrichtung einer Arbeit segnen, erbitten, erwarten' durchsetzte, in denen - nachdem sich aus dem Verbalnomen cin Infinitiv entwickelt hätte - das Objekt des Prädikatsverbums nicht das logische Subjekt des Infinitivs ist.

Eine zweite Möglichkeit der Erklärung wäre, dass der nominativische Infinitiv ursprünglich eine Lativform war; dieser Deutung fehlt jedoch aus lautlichen Gründen die Basis. ${ }^{1}$

Konstruktionen des folgenden Typs, die elliptisch anmuten, einen nominativischen Infinitiv enthalten und in Sprichwörtern und in der dichterischen Sprache bevorzugt sind, liessen sich auf Grund eines Nominativs erklären:

lov-poroškeń pramosto/ kuvaka kińeń moleme/ staka ružijań landomo 'wenn der (erste) Schnee fällt, (sollst du) auf einen langen Weg gehen, ein schweres Gewehr tragen' (='- - on pitkää tietä kulkeminen, raskasta kivääriä kantaminen'; E I 66); aul' eśinde maro čijme 'es geht nicht mit ihm zu laufen' (= 'ei (käy) hänen kanssaan juokseminen'; E III 328); kalâń kunda·mal moləoma, vettä af ṕeloma 'Wenn man auf Fischfang gehen muss, darf man das Wasser nicht fürchten' (= 'kalan pyyntiin meneminen, vettä ei pelkääminen'; M IV 695); vaj meźon tasa mod'iń érama/ vaj meźon tasa mod'iń aščma 'Ach, wie soll ich hier leben, ach, wie soll ich hier sein?' (M IV 345); koda molema täd'akaj avakaj šobdava rana jažama tšufa·ma 'Wie kann ich, liebe Mutter, früh am Morgen mahlen und zerstampfen gehen?' (M IV 549).

Obigem entspricht jedoch auch der Gebrauch des illativischen Infinitivs in den folgenden Belegen:

kolmo ugolga inińd'eńek arams,/ proruvatneńe níńd'eńek molems 'wir sollen in drei Ecken treten, wir sollen in die schluchten gehen' (E I 9); koda ńej tujems sońenze/ ḱil'd'é́

1 BCDE.vz hat diesen Infinitiv zwar mit dem v-Lativ verknüpf; Moksa- és erza-mordvin nyelvtan S. 90, 43. Vgl. PAasonen, Virittäjä 1917 s. 27. Ganz eigenartig und isoliert ist Ar LQvists Angabe über einen Infinitiv auf $-m \bar{a}$, den er Lativform nennt: Versuch einer mokscha-mordwinischen Grammatik S. 49. 
povod'eź sịnst rakšast 'Dann, als sie sich nun fortbegeben soll, sind ihre Rosse angespannt, angeschirrt' (E I 222-223); mäzaä vijsa ṕišla·dums? 'Soll ich aus aller Kraft schreien!' (E III 300 ); kuli atkazams, jofse ezdôn atkazak 'wenn dı mich verlassen willst, verlasse mich ganz' (MI IV 277 ).

Folgende Beispiele könnte man mit finn. mitä 'was', miten 'wie', millä 'womit' usw. (kann od. soll man) tehdä 'tun' vergleichen:

jomavtoms sonze, ứkaj, ńej koda·/ koda jomavtoms, ứkaj, valmavtoms 'auf welche Weise, Schwägerin, (können wir) sie töten, auf welche Weise, Schwägerin, (können wir) sie umbringen' (E I 249); a níi·ń sotsịnik, sva $\chi a \cdot m$ paro·m, níe·śt mora·ms/ a níi·ń sotsi-nilk', bojar-a·vat, ńte·śt jofta·ms 'Doch wir wissen, meine schöne Svacha, was zu singen ist, doch wir wissen, Bojarimen, was zu sprechen ist' (E II 416); kodit i mäźä monäarí tejums, duma•dnan 'Ich dachte, wie und was soll ich (nun) tun' (E III 299); aŕci- níeśt tejne er tun solle' (E III 319); boja.r kosoja·k a k'i.ŕd'i, duníl koda· bu oya·ń ratka·ms ma·ńamoń k'is 'Der Bojar konnte sich gar nicht beruhigen und sann, wie er Ocha wegen des Betruges prügeln solle' (E III 323); tence níeśt tejnems? 'Was muss man damit machen?' (E III 326); nevtiźe, kodańa ponžavtoms '(Und) er zeigte, wie man damit worfelt' (E III 326--327).

Die ursprünglichen Funktionen des illativischen Infinitivs spiegeln sich in den folgenden Beispielen wider, wo der Infinitiv die Bedeutung 'bis zu einer Handlung' hat:

eŕamozonzo ańo eŕakšnoś/ pŕaso čeŕnenze ańo šeržijams $\left(<\check{c}_{-}\right) /$kurkso pejńenze ańo ṕev́erems 'Anjuscha lebte bis ins Alter, Anjuscha, bis ihr die Haare zu ergrauen begannen, Anjuscha, bis ihr die Zähne im Munde auszufallen begannen' (E I 468); il'ado jarca vačo ṕekenk ṕeškedems/il'ado śińe čeńtań ojńenk tịń lećtams 'Esst euren leeren Magen nicht voll, trinkt eure trockene Kehle nicht feucht!' (E II 291); il'iníź kat pil'kse kaŕeń kaladoms/ kaŕce kaŕkseń śeźnevems/ lankso pal'am čełkijams/ palam artoń ńevelems 'verlasst mich nicht (so lange), bis die Bastschuhe an meinen Füssen abgenutzt sind, bis die Schnüre meiner Bastschuhe zerrissen sind, bis das Hemd auf mir staubig wird, bis mein Hemdbesatz abgeht' (E II 294); 
udomonk udoms udodo 'schlaft euren Schlaf zu Ende' (E II 476-477); netne satost ńej tone mastoroń kolems kaladoms 'das soll dir bis zum Weltuntergang genügen' (E II 507); oznịt gríiv́ennoj śv́ečań paloms, jeśli god'av́i setine či 'Sie beten, bis die 10 Kopeken-Kerze niedergebrannt ist, falls der Tag zufäliig windstill ist' (E III 25); śińenk jacams śińed'e 'trinkt, bis euer Durst gelöscht ist' (E III 161); śel'məd'ä juma·ms vanômak 'schaue mir nach, bis ich aus den Augen verschwunden bin' ( M IV 171); jar'cak topâdəms, aščak' šopâdams 'iss, bis du satt wirst, bleib, bis es dunkel wird' (M IV 220); selmestônza juma ·ms vanịnäzä 'die nach mir schaut, bis ich ausser Sicht bin' (M IV 401); mastârôń kaladâms eíäj 'lebt bis zum Untergang der Erde' (M IV 645); nu, tśora, arä sävmak kudâzt, i tŕamak patsäzen peťša.ms 'Nimm mich, junger Mann, nach deinem Hause mit und füttere mich, bis mein Flügel heil wird' (M IV $875)$.

Als eine Art Hilfsverb erscheint das unpersönliche erazi 'es ist nötig' mit dem illativischen Infinitiv. Wenn es mit dem Verb erams 'leben'l zu verbinden ist, dann würde sich der Ausdruck vielleicht ähnlich wie die Konstruktion (työ) on tehdä '(die Arbeit) muss getan werden' in einigen ostseefinnischen Sprachen erklären, z. B. estII. laud on pesta 'der Tisch ist zu waschen, muss gewaschen werden'. ${ }^{2}$

éavat, kakaj, urvakstoms 'man sollte dich, Kind, verheiraten' (E I 502); vešriems erati alganžej-tikšet 'Man muss alganžejKräuter suchen' (E 1 Il 166); sịnst éaći kočkams i kośtams. setne ejse éa $\because$ suftams 'Man muss sie sammeln und trocknen. Mit ihnen muss man räuchern' (E III 167); olknịn éäíit' śembiiń vačkams usfs 'Das Stroh muss alles aufgeladen werden' (E III 301); ala-šat́ne era ${ }^{2} i$ ando $m s$ 'Man müsse die Pferde füttern' (E III 319); éäuvi eśte-inza piidams 'er muss selbst kochen' (M IV 823).

Ein solcher Ausdruck wie (nurda naäjat, ičk’źi) araj moləms '(Siehst du einen Schlitten,) sollst du (weithin) fahren' (M IV 734) könnte wiederum mit dem finnischen tulee mennä 'soll

I Pansonen, Mordw. chrestomathie S. 63; Ahlqvist, a.a.O. S. 88, 42.

2 Saukkonen, erw. Manuskript S. 162. 
grehen' (arams 'sich stellen, hintreten; werden, sich verwandeln') verglichen werden. Die entsprechende Konstruktion mit einem nominativischen Infinitiv mońdein araj kulâma 'muss ich sterben' (M IV 809-810) lässt sich leicht durch den Nominativ erklären: 'mir kommt das Sterben'.

Als unpersönliches Hilfsverb wird mit dem illativischen Infinitiv auch das russische Lehnwort nel'za 'man darf nicht, man kann nicht, es ziemt sich nicht, es ist nicht möglich, ist unmöglich' gebraucht:

uk, avakaj d'írinem,/ nelźa mońde a molems 'Oh, Mutter, meine Ernährerin, ich kann nicht anders als Gehen' (E I 23); uk nel źa liśems tenze tuŕmasto 'Er kann nicht aus dem Kerker entkommen' (E I 194); nel'za teń liśems jalga jutks 'ich darf nicht zu Freundinnen hinausgehen' (E I 287); uk nelźa liśems tejteŕne jalga jutks 'Das Mädchen darf nicht mit den Freundinnen ausgehen' (E I 477); kudov saś pop, nelźa i kortams teńende 'Der Pope kam nach Hause, und er durfte (einfach) nicht sprechen' (E III 339).

Der illativische Infinitiv hat mitunter wie ein Attribut gebraucht werden können:

raužo biśor surgutoń pakśań sokams-modazo '(Wie aus) schwarze(n) Perle(n) ist die Pflugerde der Mark Surgut' (E I 471); da lamo sonze surgutoń śtepkeń led'ems lugazo 'die Steppe Surgut hat viel Wiese zum Mähen' (E I 472); uk ṕiže čuŕka ledems tikšezę uš śijań blida śimdams ŕed'eze '(wie) grüne Zwiebeln ist ihr zu mähendes Gras, (wie) eine Silberschüssel (glänzt) ihr Trinkwasser' (E I 150).

Im folgenden tritt auch der nominativische Infinitiv parallel als Attribut auf:

suka-valnẹs maŕav́i/ k'iŕgava kajams/ jakśtere ḱel'ej l'entučka/ kurva-valneśs maíavii/ ṕeŕka karksamo/ sẹ́rej-alga zapunkaks 'das Wort "Hündin" kommt mir vor wie ein um den Hals zu werfendes rotes, breites Band, das Wort "Hure" kommt mir vor wie eine umzubindende Schürze mit breiter Verbrämung am unteren Rand' (E II 282).

Das russische Lehnwort davaj wird anf die gleiche Weise mit beiden Infinitiven verwendet:

"dava·j", meŕi, "ńeń v́e·jse era·mo!» "Lass uns nun zusam8- Finnisch-ugr'sche Forschungen 
menleben", sagte er!' (E III 216); ćora·ś k'eži·jaś koźe·jkaśt lạks i dava $j$ čavmo. esnẹ.nde 'Der Mann wurde auf die Gattin böse und begann sie zu schlagen' (E III 265); davaj čijme, kona śed'e bojka 'Lass uns laufen wer schneller ist!' (E III 328); tava.j jalgaj moratama 'Lass uns singen, Freund' (M IV 424).

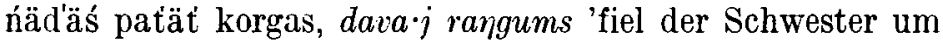
den Hals und begann zu weinen' (E III 273); di popoś ńiéri: davaj zavtrokams 'Und der Pope sagte: "Lass uns frühstücken!"' (E III 312); davaj, settnen esa ńef tams 'Er begann geradeswegs an den Dielen zu kratzen' (M IV 813); i purômśt ṕérfkanza śemb́ä tuvôtńä i davaj íešandəms sojń 'und alle Schweine sammelten sich um es herum und begannen ihn zu suchen' (M IV 815).

Abschliessend einige Beispiele von Formen, die neben dem normalen illativischen Infinitiv auftreten und den Bindevokal bewahrt haben sowie vom Translativ des Infinitivs, der in der gleichen Funktion verwendet wird:

ruzńi kunda·źä kulâms šavâmas 'der Russe aber packte sie, um (sie) totzuschlagen' (= 'tarttui kiinni lyödäkseen kuolemaan'; M IV 109); əl'i al’äçəń jar'cams kšrna c soń ajaš,/ əl'i d'äjaćəń śimmâs pojlañac ajaš? 'Hat dein Vater kein Brot zu essen, oder hat deine Mutter kein Getränk zu trinken?' (M IV 368); kati vajmams, kati tumâs tanga šl'adndama 'Sollte man ruhen oder wieder herumschlendern gehen?' (M IV 829); śiń dava·j śimmâs jar'tsams 'Sie fingen an zu trinken und zu essen' (M IV 832); de·inza (< sońd'e-inza) aš meźä tijmâs, araj, mäŕkś mol'mâs 'Es ist nichts zu machen. "Man muss wohl hingehen", sagte er' (M IV 834); iliníiź uče pil'kse kaŕeń kaladmos/ iliníiź uče kaŕksen śeźnevmes 'wartet nicht auf mich, bis die Bastschuhe an meinen Füssen abgenutzt sind, wartet nicht auf mich, bis die Schnüre meiner Bastschuhe zerrissen sind' (E II 462).

konań kavto a ńej ćora ejd'enze/ v́ejkeń sajmeks ińazoro ńej pazne 'wer zwei Söhne hat, (dem) soll der eine für den Kaiser genommen werden' (E I 465; vgl. konań kavto ulit ćorakakanzo/ v́ejkeń sajems ińazoro ńej paznẹ 'wer zwei Söhne hat, (dem) soll der eine für den Kaiser genommen werden'; E I 461); araś, tirí av́ińem,/ liśmeks sovamks kenkšinet'/ pañzmoks śolgomks valminet/ kuźmeks ṕećka-lanǵinet' 'Du hast, meine liebe 
Mutter, keine Tür um hinauszugehen und hereinzukommen, kein Fenster um es zu öffnen und zu schliessen, keinen Ofenplatz

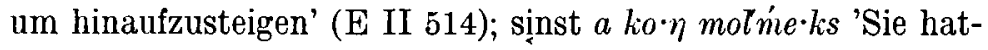
ten nichts, wohin sie gehen konnten' (E III 215); ali jar'tsams kšizä ajaš/ ali simemeks pojlazä aš./ ali štšamâks platäzä aš? 'Oder habe ich nicht Brot zu essen, oder habe ich nicht Getränk zu trinken, oder habe ich nicht Kleider anzuziehen?' (M IV 149); ali kšinä·tsä polanäj jar'tsamks ajaš/ ali salnätsä polanäj langāzônza kajams toń ajaš? 'Oder hast du, Mann, kein Brot zu essen, oder hast du kein Salz, Mann, es darauf zu schütten?' (II IV 333). ${ }^{1}$

1 S. auch Очерки мордовских диалектов S. 56.

Pauli Saukkonen 